\title{
AS-BUILT RELIABILITY IN ARCHITECTURAL HBIM MODELING
}

\author{
P. Maiezza ${ }^{1}$ \\ ${ }^{1}$ Department of Civil, Construction-Architectural and Environmental Engineering, University of L'Aquila, L'Aquila, Italy, \\ pamela.maiezza@graduate.univaq.it
}

Commission II

KEY WORDS: architectural heritage, HBIM, As-Built, reliability

\begin{abstract}
:
The As-Built Model cannot be considered as a simple three-dimensional mould of the studied reality but as a process of analysis, synthesis and communication of architectural complexity including, in addition to geometric-dimensional aspects, also the historical, aesthetic and architectural features of the building.

Consequently, the transparency and reliability issues of the digital visualization constitutes, as well as for the field of archaeology, a matter of primary importance in the modelling of cultural heritage.

The increasing interest of scholars in the application of Building Information Modelling (BIM) to historical buildings has renewed the problems related to the reliability of the As-built, related not only to the relationship between the model and the measure, but also to the other information, for example the constructive technologies.

Based on the survey and modelling of some case studies, the paper's aim is to define a reference standard for the reliability declaration of the As-Built HBIM models, which considers both the geometric and information aspects.
\end{abstract}

\section{INTRODUCTION}

In the case of historical architecture, the concept of "As-Built" model, often limited to geometric-dimensional aspects, cannot fail to take account historical, material, constructional characteristics, degradation, etc.

All these aspects are the result of critical study processes, referring to non-homogeneous sources, direct and indirect (diagnostics, archival documentation, etc.), for each of them it is appropriate to indicate an "interpretative" level of the information derived from the available data.

The issue of the model's "Transparency" becomes essential, understood as the declaration of the information sources and the possibility of philological reconstruction of the choices made for the realization of the As-Built model (The London Charter, 2009; Principles of Sevilla, 2012).

The principle of transparency has been widely developed in the archaeological field, where the digital reconstruction of artefacts, in their ancient configuration, is largely based on indirect information, comparative analysis, interpretative hypothesis (Brusaporci, 2017a).

Also of critical importance is the theme of "Reliability" of the model compared to the reality.

It is usually interpreted as a deviation of the model compared to the point cloud, but the concept can also be extended to other information content (De Luca, 2011; Apollonio, 2015; Brusaporci, 2017b) (Figure 1).

Building Information Modelling, as an informative procedure specifically dedicated to architecture, allows the management of data that go beyond the only geometric-dimensional aspect.

In its application to the built heritage - theme still widely open (Bianchini et al., 2017) -, the Building Information Modelling highlights the problems of reliability, related not only to the relationship between the model and the measure (Apollonio et al. 2013; Quattrini et al. 2016), but also to the other information such as the constructive technologies (Brusaporci et al. 2018).

The paper deals with the transparency and reliability issues for the architectural heritage modelling within the BIM environment. In particular, on the basis of the survey and modelling of some case studies, the paper's aim is to define a reference standard for the reliability declaration of the As-Built HBIM models.

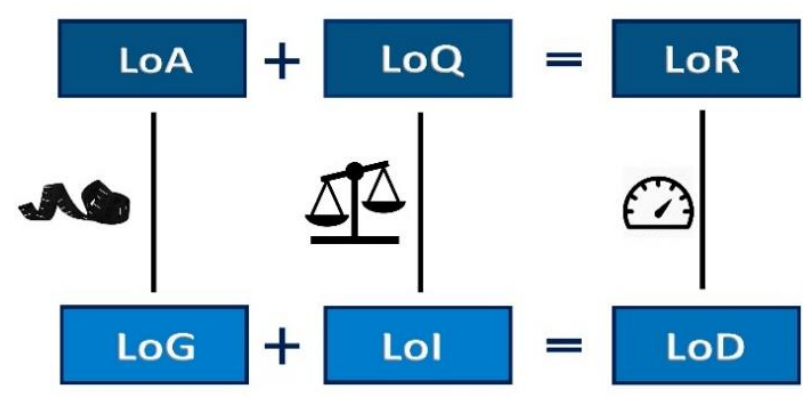

Figure 1: The model's reliability is the synergy of the geometric accuracy (LoA) and the quality of information sources (LoQ).

\section{TRANSPARENCY AND RELIABILITY}

Among the sectors involved in the built heritage, archaeology first investigated digital visualization as a research methodology, putting the philological interpretation issue of the reconstructive models of artefacts no longer existing and reconstructed on the basis of partial archaeological findings, news literary studies, typological studies (Frischer, 2008; Greengrass and Hughes, 2008).

In the modelling and digital visualizations of the built heritage, the concept of "transparency" of the virtual reconstruction becomes central.

Basically developed by archaeology scholars, although generally referring to computer based visualization, essential reference for the definition and understanding of transparency is The London Charter (2009). Subsequently, Principles of Sevilla (2012) were written specifically for the archaeological field.

The London Charter defines "Intellectual transparency" as: "The provision of information, presented in any medium or format, to allow users to understand the nature and scope of "knowledge claim" made by a computer-based visualization outcome" (p.12). The Principles of Seville, introducing the "Scientific Transparency", they state that: "All computer-based visualization must be essentially transparent, i.e. testable by other researchers or professionals, since the validity, and therefore the scope, of the conclusions produced by such visualization will depend largely 


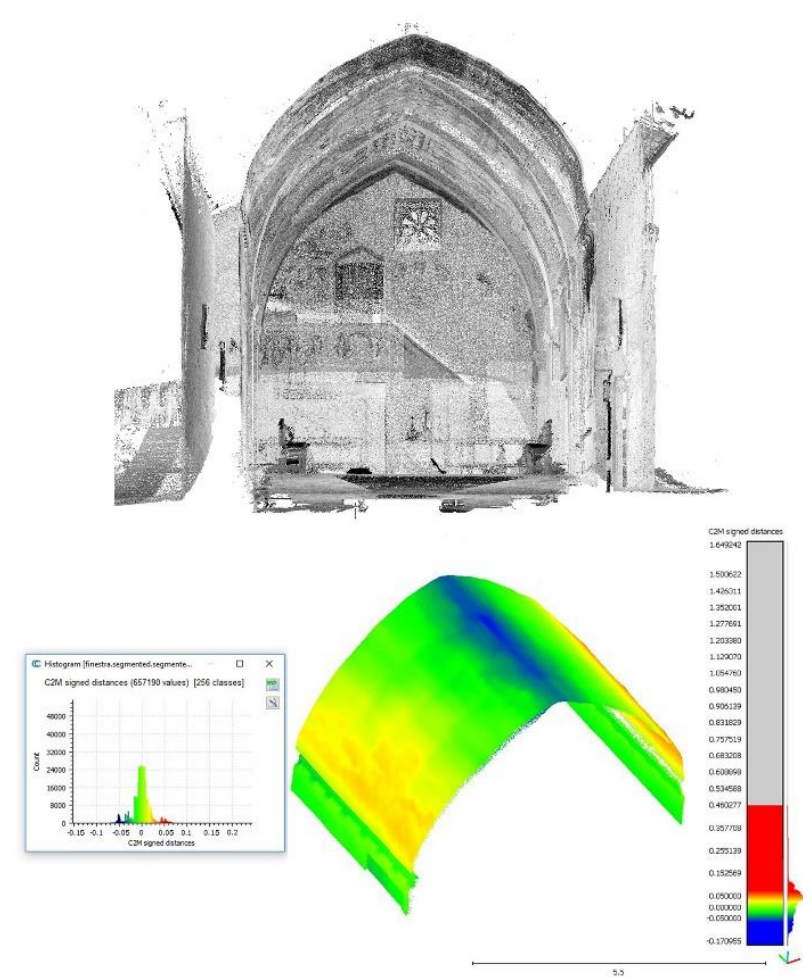

Figure 2: Evaluation of the Level of Accuracy (LoA): deviation between the point cloud and the model of a portion of San Pellegrino church's vault in Bominaco (L'Aquila) (13th century).

on the ability of others to confirm or refute the results obtained" (p.8).

A fundamental tool for achieving transparency is the paradata, defined by The London Charter as "Information about human processes of understanding and interpretation of data objects. Examples of paradata include descriptions stored within a structured database of how evidence was used to interpret an artefact, or a comment on methodological premises within a research publication. It is closely related, but somewhat different in emphasis, to "contextual metadata", which tend to communicate interpretations of an artefact or collection, rather than the process through which one or more artefacts were processed or interpreted" (p. 13).

In particular "Paradata may be seen as a digital equivalent to "scholia", as well as an addition to the traditional critical apparatus for describing the process of reasoning in scholarly research" (Bentkowska-Kafel et al.,2012) (p.2).

Paradata is a kind of metadata focused on the use of data, «but the big difference to keep in mind is that paradata involves "action" and traditional metadata involves "classification" (US Department of Education, 2011) (p1).

Paradata is not only about the use of metadata, but also describes the transformation of data during the digital reconstruction process.

Certainly, the extension of the concept of transparency from the field of archaeology to that of architecture requires specific reflections (Brusaporci and Trizio, 2013).

Historic buildings have different characteristics compared to other types of built heritage, both in terms of the material, historical, constructive, technological aspects and the documentation available. Moreover, they are buildings that are mostly used daily, subject to processes of modification and stratification over the centuries, witnesses of the cultures that have followed one another over time.
Every architectural representation, both of the current state of a building and of a past configuration or of a never realized one, constitutes an interpretation, the result of a critical reading made by the scholar.

The As-Built Model, therefore, is not to be taken as a simple three-dimensional mould of the investigated reality, but as a process of analysis, synthesis and communication of architectural complexity, including, beyond the geometric-dimensional aspects, also the historical, aesthetic and architectural features of the artefact.

Consequently, the subject of the transparency of digital visualization constitutes, as well as for the field of archaeology, a issue of primary importance.

The importance of the transparency and reliability issues in the field of architectural heritage modelling has led to the development of interesting studies, mainly focused on the procedures for declaring the level of reliability of the representation in relation to the type of source used.

Borra (2014), considering the $3 \mathrm{D}$ model as an interface to communicate the work methodology, proposes four levels of coherence of the model's shape: The reliability according to "objective" data (also derived from documental information); The reliability according constructive analysis; The coherence to architectural styles; Interpretative hypothesis. Another question is about the comparison between the representation of different reconstructive hypothesis. This highlights the interpretative nature of 3D model.

Stefani et al. (2010) focus on temporal uncertainty of historical transformations of the building. Semantization and model's "granularity" allow the representation of spatial and temporal uncertainly, according to simultaneous windows with 3D views, graphs and datasets.

De Luca et al. (2011) propose geometric elements distinguished by colour tones concerning spatial (shape or position) and temporal uncertainly (dating). About building transformations, authors describe changes with graphic notations (creation, destruction, alteration, union, division, reconstruction), related to historical visualizations. About geometric restitutions, four levels of reconstruction are defined: 1) based on surveying data; 2) based on images in conic or cylindrical projection; 3) based on images in pseudo-perspective, pseudo-axonometry or sketches; 4) without iconographic support or survey data.

Apollonio et al. (2013) deal with the issue of "uncertainly" of 3D models of no longer existing or never realized building. They propose an uncertainty gradient colour code, referred to the kind of information source: Original drawing; Original sketch; Coeval design reference; Architect's treaties or books; Treaties references; Architectural styles; Construction system; Failing references. It's evident that this approach is related to the characteristics of the case study: i.e. only planned villas, not existing.

Considering different kinds of architectural heritage, in Apollonio e Giovannini (2015), reliability is defined through a gradient colour scale related to the different sources: Laser scanning survey; Original drawings; Original sketches and uncomplete survey drawings; Coeval design reference (same architect and way of representation); Data deducted from previous levels; Failing references.

In order to ensure the transparency of digital visualization, Brusaporci (2017b) considers the declaration of the sources on which the construction of the model is based, both architecturally and constructively, and the description of their critical interpretation (extrinsic paradata) to be fundamental. This is combined with the importance of the information regarding the modelling process from an instrumental point of view (intrinsic paradata). 
In order to guarantee the scientific rigor and transparency of the interpretative processes of architectural heritage in BIM environment, Bianchini et al. (2018) introduce the Level of Reliability (LOR) as a new attribute in support of digital objects. This parameter, with a value between 0 and 10, is the result of the average of the various factors that can affect both the element's geometric reliability and the ontological correspondence between the virtual object and the real one.

For a more immediate declaration of the model's reliability, moreover, the numerical scale of the LOR is translated into a simplified chromatic scale, with only three levels of reliability (high, medium and low).

Finally, Brumana et al. (2018) define modelling protocols, called "Grades of Generation" (GoGs), aimed at obtaining a Level of Geometry -in reference to the subdivision of the LoD in LOG and LOI made by the UNI 11337 - coherent to the accuracy obtained from the survey.

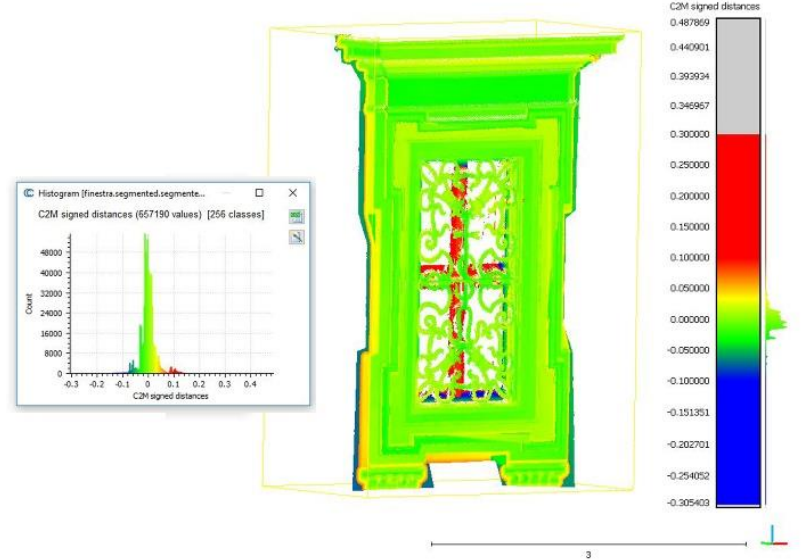

Figure 3: The deviation analysis of a window of St. Basilio monastery in L'Aquila (19th century) for the Level of Accuracy (LoA) evaluation.

\section{HBIM AND AS-BUILT}

The potential offered by BIM in the management of processes related to new buildings, are also of great interest for the specific needs of the architectural heritage, despite the inherent difficulties of extending the BIM approach to historic buildings. Hence the wide diffusion of studies dedicated to it (Murphy et al., 2011; Apollonio et al., 2012; Garagnani, 2013; Dore and Murphy, 2015).

The modelling in the BIM environment, specifically designed for architecture, is based on the use of semantically recognized 3D objects that, in addition to their geometric characteristics, contain all the aspects that distinguish the real architectural element, including the construction technologies.

It is evident, therefore, especially to BIM, the issues of transparency and reliability of digital visualization can not stop at the geometric-dimensional component but must include all the aspects regarding the knowledge of a historical building. Therefore, the theme of deviation, intended as the difference between the model and the real object represented by the point cloud, is accompanied by reflections related to the other information content of the model (Figure 2).

In this respect, it is recalled the recent UNI 113337 standard: according to the English system (PAS 1192-2 of 2013) and the American system (BIMForum), the LODs are defined on the basis of both the levels of development of the graphic attributes (level of object development - geometric attributes (LOG)) and non-graphic ones (level of object development - information attributes (LOI)).

If the declaration of tools, use, modelling process, etc., is an essential aspect for the transparency of each digital visualization, this assumes an even more marked value in the case of BIM, an approach that, designed for the project of the new buildings, is still failing to fully meet the specific needs of architectural heritage.

The difficulties in reconciling the use of standardized libraries with complex and unique architectural elements, not included in the current software (for example vaults, irregular masonry walls, etc.), requires the adoption of specific and solutions (Oreni et al., 2014), whose declaration is of fundamental importance for the transparency of the process of interpretation and representation.
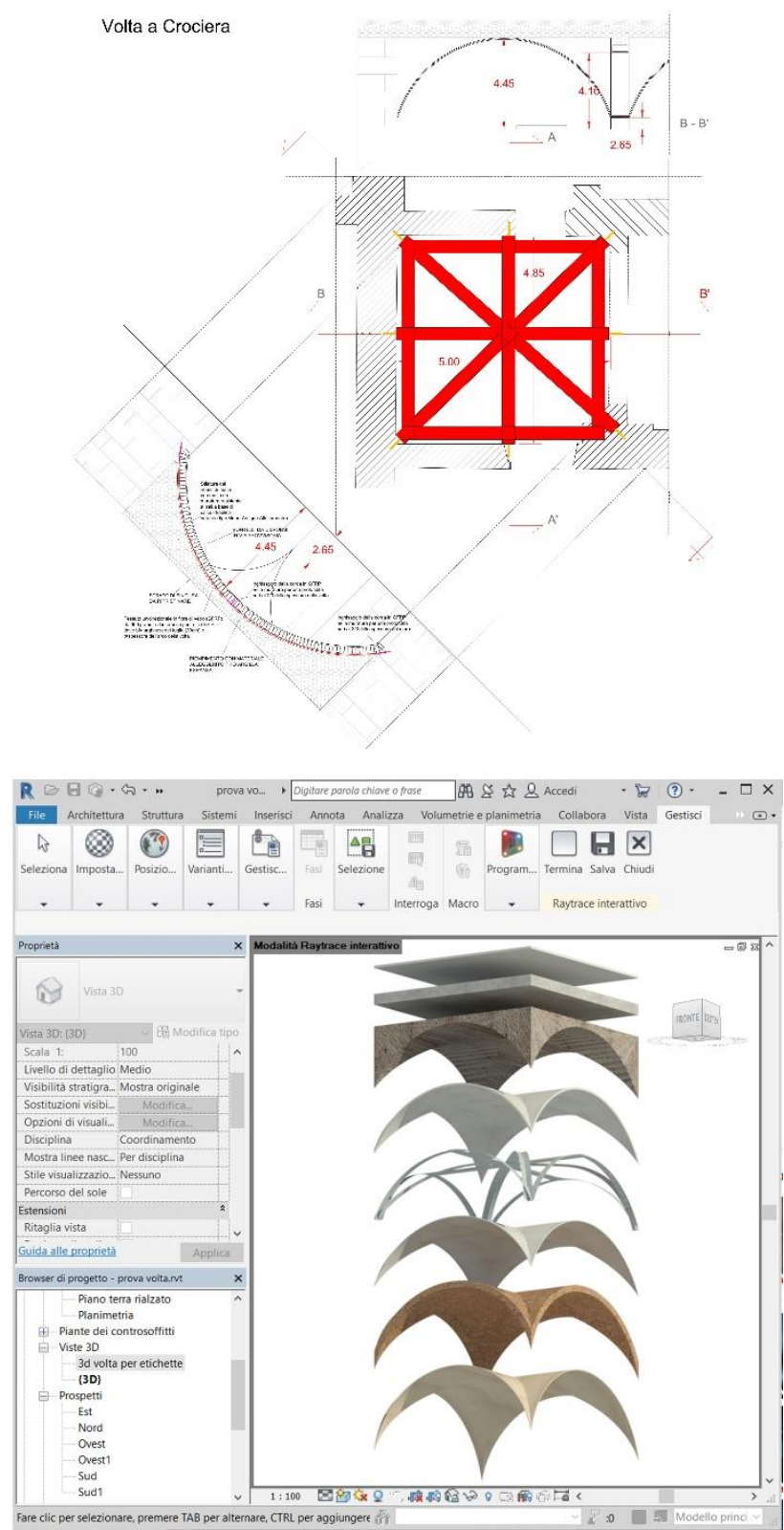

Figure 4: The model of a vault of Palazzo Camponeschi in L'Aquila (17th century) and the sources of information for the modelling of its construction technologies: example of Level of Quality (LoQ). 


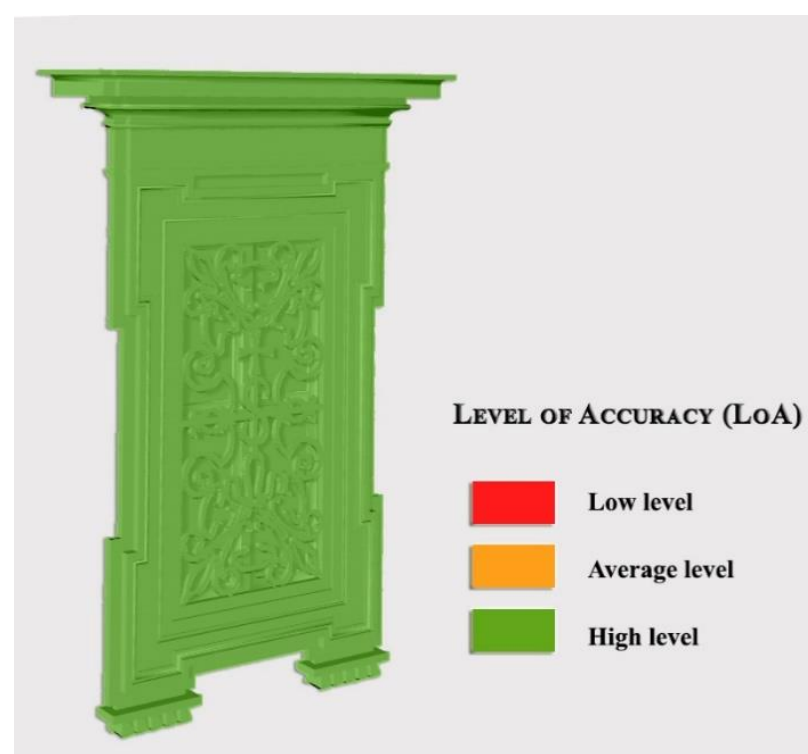

Figure 5: Level of Accuracy (LoA) of the model of St. Basilio's window: High LoA, that is $70 \%$ of the model has a deviation less than $20 \mathrm{~mm}$, and $30 \%$ with a deviation less than $50 \mathrm{~mm}$

\section{A PROPOSAL OF STANDARDIZATION}

In view of the dual nature of the BIM (model and database) and the subdivision of the "Level of Development of the object" (LoD) in LOG (Level of Development of the object GEOMETRIC ATTRIBUTES) and LOI (Level of Development of the object - INFORMATION ATTRIBUTES), made by the UNI 11337: 2017 standard, a double level of reliability is proposed for digital objects:

- Level of Accuracy (LoA), relating to the geometric accuracy, measured as deviation (Figure 3);

- Level of Quality (LoQ), concerning the information content associated with the single element (Figure 4).

The two levels, together, measure the reliability of the architectural element model (Level of Reliability - LoR).

BIM is not provided for paradata, however its dual value of graphic representation, on the one hand, and informative content, on the other, can be exploited to record and communicate data, information and interpretative choices that underlie the modelling.

In particular, the possibility of storing and managing a large quantity of heterogeneous data offered by BIM can be used to link to the different components of the model the documents (images, surveys, historical photos, etc.) that have led to their construction. to declare the informative framework underlying the modelling of the historical building.

Since attributing to the whole model a single LoR, result of the average of the individual levels, would indiscriminately accumulate objects characterized by an abundant availability of information to elements characterized by rather incomplete knowledge, it is considered appropriate to refer the Level of Reliability to the individual semantic components.

The evaluation of the deviation between the building model and the high resolution detail provided by the massive 3D acquisition systems has different connotations according to the type of virtual reconstruction. This is because the modelling and, therefore, its reliability is strictly dependent on the objectives, as also happens in the architectural survey. The same connection with the purposes is found in the reliability of not geometricdimensional information.

Beyond the modelling purposes, the definition of reliability range must take into account two other factors: the characteristics of the historical building, because a greater geometric and architectural complexity directly affects the construction of the model and the reliability of its components; the Level of Development of the model.

Just like the survey is linked to the scale of representation, in fact, modelling depends on the level of definition to be achieved.

The different degree of infographic definition of the model entails a different level of reliability: with the same information available on a historical building, the reliability of the model is greater for a non-advanced geometric and informative detail, i.e. for a lower level of development. As the LOG and LOI progress, the model's level of reliability decreases if there is not a corresponding increase in geometrical accuracy, data and information to support the creation of digital elements.

Based on the analysis of some case studies, we propose a standardization of the reliability statement - understood as the synergy of LoA and LoQ - according to three different levels: high, medium, low.

As regards the geometric reliability (LoA), moving from the comparison between the models' accuracy and the point clouds' detail, three levels have been established corresponding to three deviation ranges relative to percentages of the model's surface (Figure 5). In particular, on the basis of experimentations carried out and also with reference to the study by Brumana et al. (2018) and to the definition degree usually adopted in the professional practice, we have:

- Low level for $70 \%$ of the model's surface having a deviation greater than $50 \mathrm{~mm}$;

- Average level when $70 \%$ of the model has a deviation value between $20 \mathrm{~mm}$ and $50 \mathrm{~mm}$, and $30 \%$ with a deviation greater than $50 \mathrm{~m}$;

- High level when $70 \%$ of the model has a deviation less than $20 \mathrm{~mm}$, and $30 \%$ with a deviation less than $50 \mathrm{~mm}$.

In the case of an inadequate Level of Accuracy, the model must be adjusted until it meets minimum requirements, according to an iterative process. In this regard, it is recalled how HBIM modelling can also include NURBS surfaces or meshes imported from other applications (Oreni et al., 2014), in order to model complex geometrical shapes.

The model's reliability related to its information content (LoQ) is linked, instead, to the sources used.

In particular, we have:

- Low reliability level for the exclusive use of indirect sources, i.e. derived from other sources, which allow only a reconstructive hypothesis of the object;

- Average reliability level for the use of primary sources (directly concerning the analysed architectural element) not exhaustive, since they leave open doubts and uncertainties;

- High reliability level in the case of a modelling based on the use of direct sources, which allow a complete knowledge of the architectural element.

Moreover, since the model's informative contents can be of a profoundly different nature (historical, relating the construction technologies, etc.), the Level of Quality concerns the individual contents, leading to a sort of sub-reliabilities. For example, there will be a LoQ concerning the reconstruction of previous historical phases and one the constructive system (Figure 6).

Both Levels of Reliability of digital objects - both geometric and inherent information attributes - are expressed with colour tones by using visualization filters, which allow immediate communication and comprehension.

Finally, in consideration of the gradualness of the knowledge process of a historical building, a progressive path that persists even during the restoration building site, this dual level of reliability is not to be considered immutable but variable according to the knowledge advancing. 


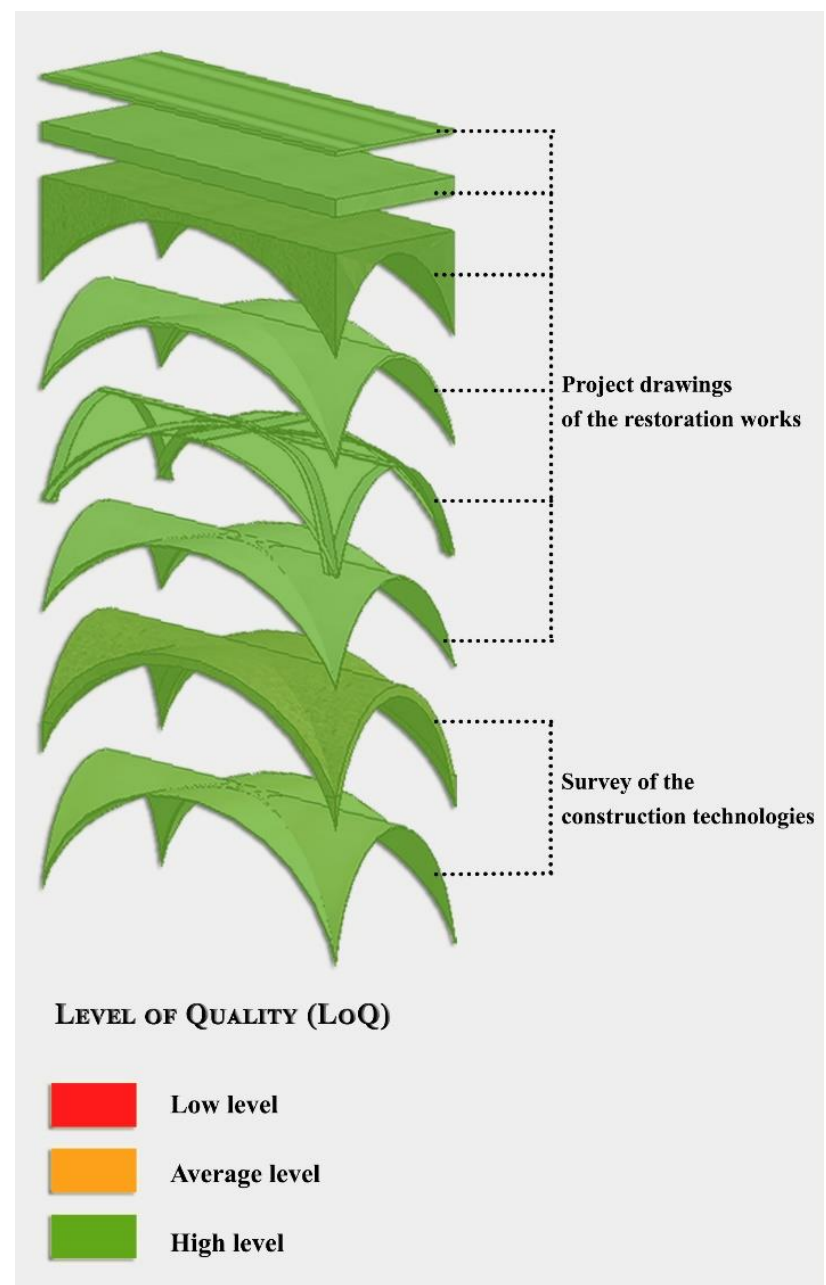

Figure 6: Level of Quality (LoQ) of the model of a vault of Palazzo Camponeschi: High reliability level for the construction technologies since the modelling has been completely based on the use of direct sources (survey and visual inspection permitted by restoration work; project drawings of the restoration works).

\section{CONCLUSIONS}

In the field of digital visualization of architectural heritage, the theme of transparency and reliability is of central importance to ensure the reproducibility and testability of the models by other scholars and, therefore, the scientific nature of the threedimensional representation.

The declaration of the sources and a critical evaluation of the representation based on them is an essential aspect for the study and knowledge of the architectural heritage.

In conclusion, citing the well-known DIKW hierarchy, i.e. Data - Information - Knowledge - Wisdom hierarchy (Ackoff, 1989), the process of knowledge and modelling of an architectural asset can be summarized as follows: the critical analysis of the data coming from the different types of source (direct or indirect) allows to produce information represented by digital objects characterizing by specific LoD; the assessment of the modelling reliability leads to the knowledge of the historical building and, hence, to the ability to develop what has been learned and elaborated, namely the "wisdom" represented by the As Built BIM model (Figure 7).

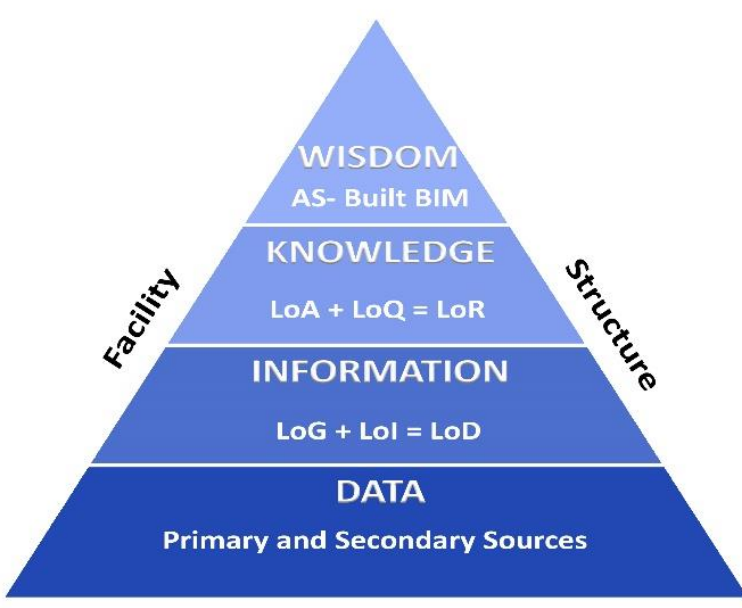

Architecture

Figure 7: The DIKW hierarchy and the process of knowledge and modelling of an architectural asset.

\section{ACKNOWLEDGEMENTS}

The research has received funding from the Italian Government under Cipe resolution n.135 (Dec. 21, 2012), project INnovating City Planning through Information and Communication Technologies (INCIPICT).

\section{REFERENCES}

Ackoff, R.L., 1989. From Data to Wisdom. Journal of Applied System Analysis, 16, 3-9.

Apollonio, F.I., Gaiani, M., \& Zheng, S., 2012. BIM-based modeling and data enrichment of classical architectural buildings. SCIRES-IT, 2 (2).

Apollonio, F.I., Gaiani, M., and Sun, Z., 2013. Characterization of Uncertainty and Approximation in Digital Reconstruction of CH Artifacts. In: Proceeding of Le Vie dei Mercanti XI Forum Internazionale di Studi. Napoli: La Scuola di Pitagora, pp.860869.

Apollonio, F.I., and Giovannini, E.C., 2015. A paradata documentation methodology for the Uncertainty Visualization in digital reconstruction of $\mathrm{CH}$ artefacts. SCIRES-IT, 5(1), 1-24.

Bentkowska-Kafel A., Denard H., and Baker D., 2012, eds. Paradata and Transparency in Virtual Heritage. Farnham: Ashgate Publishing.

Bianchini, C., Inglese, C., Ippolito, A., Maiorino, D. and Senatore, L. J., 2017. Building Information Modeling (BIM): Great Misunderstanding or Potential Opportunities for the Design Disciplines?. In: Ippolito, A., Cigola, M., eds., Handbook of Research on Emerging Technologies for Digital Preservation and Information Modeling. Hershey, PA: IGI Global, pp. 67-90. 
Bianchini, C., Nicastro, S., 2018. The definition of the Level of Relability: a contribution to the transparency of Historical-BIM process. In: 3D Modeling \& BIM, pp. 208-225.

Borra, D., 2004. Sulla verità del modello 3D. Un metodo per comunicare la validità dell'anastilosi virtuale. In. Malinverni, E.S, ed., Proceeding of eArcom04 Tecnologie per comunicare l'architettura. Ancona: CLUA, pp. 132-137.

Brumana, R., Della Torre, S., Previtali, M. et al., 2018. Generative HBIM modelling to embody complexity (LOD, LOG, LOA, LOI): surveying, preservation, site intervention - the Basilica di Collemaggio (L'Aquila). Appl Geomat (2018) 10: 545. https://doi.org/10.1007/s12518-018-0233-3.

Brusaporci, S, Trizio, I, 2013. La "Carta di Londra" e il Patrimonio Architettonico: riflessioni circa una possibile implementazione. SCIRES-IT, Volume 3, Issue 2 (2013), pp.5568.

Brusaporci, S., 2017a. Digital Innovations in Architectural Heritage Conservation: Emerging Research and Opportunities. Hershey, PA: IGI Global

Brusaporci, S., 2017b. The Importance of Being Honest: Issues of Transparency in Digital Visualization of Architectural Heritage. In: Ippolito, A., ed., Handbook of Research on Emerging Technologies for Architectural and Archaeological Heritage. Hershey, PA: IGI Global, pp. 66-93.

Brusaporci, S., Maiezza, P., and Tata, A., 2018. A FRAMEWORK FOR ARCHITECTURAL HERITAGE HBIM SEMANTIZATION AND DEVELOPMENT. In: Int. Arch. Photogramm. Remote Sens. Spatial Inf. Sci., XLII-2, 179-184.

De Luca, L., Bussayarat, C., Stefani, C., Véron, P., and Florenzano, M., 2011. A semantic-based platform for the digital analysis of architectural heritage. Computers \& Graphics, 2(35), 227-241.

Dore, C., \& Murphy, M., 2015. Historic Building Information Modelling (HBIM). In: Brusaporci, S., ed., Handbook of Research on Emerging Digital Tools for Architectural Surveying, Modeling, and Representation. Hershey, PA: IGI Global, pp. 233-273.

Ente Italiano di Normazione, 2017. Gestione digitale dei processi informativi delle costruzioni, UNI 11337:2017.

Frischer, B., 2008. From digital illustration to digital heuristic. In B.D. Frischer, ed., Beyond illustration: $2 d$ and $3 d$ Digital Technologies as Tool for Discovery in Archaeology. Oxford: British Archaeological Reports.

Garagnani, S., 2013. Building Information Modeling and real world knowledge: a methodological approach to accurate semantic documentation for the built environment. In: Proceedings of Digital Heritage 2013, vol.1 (pp.489-496). Marseille: IEEE.

Greengrass, M., \& Hughes, L. (Eds.) (2008). The Virtual Representation of the Past. Farnham: Ashgate.

Murphy, M., McGovern, E., \& Pavia, S., 2011. Historic building information modelling - adding intelligence to laser and image based surveys. In: International Archives of the Photogrammetry, Remote Sensing and Spatial Information Sciences, XXXVIII5/W16.

Oreni, D., Brumana, R., Della Torre, St., Banfi, F., Barazzetti, L., and Previtali, M., 2014. Survey turned into HBIM: the restoration and the work involved concerning the Basilica di Collemaggio after the earthquake (L'Aquila). In: ISPRS Annals of the Photogrammetry, Remote Sensing and Spatial Information Sciences, Vol. II, 2014, pp. 267-273.

Principles of Sevilla, 2012, http://smartheritage.com/sevilleprinciples/seville-principles (Last accessed on July 2018).

Quattrini, R., Clini, P., Nespeca, R., and Ruggeri, L., 2016. Measurement and Historical Information Building: Challenges and opportunities in the representation of semantically structured 3D content. DISEGNARE CON vol. 9 n. 16, 2016, p. 14.1-14.11.

Stefani, C., De Luca, L., Véron, P., \& Florenzano, M., 2010. Time indeterminacy and spatio-temporal building transformations: An approach for architectural heritage understanding. In: International Journal on Interactive Design and Manufacturing, 4(1), 61-74.

The London Charter (2009), http://www.londoncharter.org/ (Last accessed on July 2018).

US Department of Education (2011). Paradata in 20 Minutes or Less,

https://docs.google.com/document/d/1QG01AmJ0ztHJq5DbiTG Qj9DnQ8hP0Co0x0fB1QmoBco/edit?pli=1 (Last accessed on December 2018). 